\title{
Redescription of Sarcocystis fusiformis sarcocysts from the water buffalo (Bubalus bubalis)
}

\author{
J. P. DUBEY ${ }^{1}$, M. HILALI ${ }^{2}$, E. VAN WILPE ${ }^{3}$, S. K. VERMA ${ }^{1}$, R. CALERO-BERNAL ${ }^{1}$ \\ and $\mathrm{A}$. ABDEL-WAHAB ${ }^{2}$ \\ ${ }^{1}$ U. S. Department of Agriculture, Agricultural Research Service, Beltsville Agricultural Research Center, Animal Parasitic \\ Diseases Laboratory, Building 1001, Beltsville, Maryland 20705-2350, USA \\ ${ }^{2}$ Parasitology Department, Faculty of Veterinary Medicine, Cairo University, 12211 Giza, Egypt \\ ${ }^{3}$ Department of Anatomy and Physiology, Faculty of Veterinary Science, University of Pretoria, Onderstepoort 0110, \\ South Africa
}

(Received 8 May 2014; revised 11 Fune 2014; accepted 16 Fune 2014)

\begin{abstract}
SUMMARY
Four valid species of Sarcocystis have been reported from the water buffalo (Bubalus bubalis): Sarcocystis fusiformis, Sarcocystis buffalonis, Sarcocystis levinei and Sarcocystis dubeyi. Here, we redescribe structure of S. fusiformis sarcocysts by scanning and transmission electron microscopy (SEM, TEM). Twenty-one macroscopic sarcocysts from oesophagus of the water buffalo in Egypt were examined by light microscopy, SEM and TEM. The sarcocyst wall was up to $9 \mu \mathrm{m}$ thick, depending on the section and the technique. In $5 \mu \mathrm{m}$ paraffin-embedded sections, the sarcocyst wall was indistinct, 2-5 $\mu \mathrm{m}$ thick and appeared smooth. In $1 \mu \mathrm{m}$ plastic-embedded sections stained with toluidine blue, the sarcocyst wall was $2 \cdot 5-5 \cdot 2 \mu \mathrm{m}$ thick and had branched villar protrusions (vp)-like branches of a dead tree. By SEM, the sarcocyst wall had a mesh-like structure with irregularly shaped vp that were folded over the sarcocyst wall. On each vp there were uniform papillomatous structures that were $100 \mathrm{~nm}$ wide. By TEM, vp were up to $6 \mu \mathrm{m}$ long and contained filamentous tubular structures, most of which were parallel to the long axis of the projections; granules were absent from these tubules. By TEM, bradyzoites within the same cyst varied from $11 \cdot 2$ to $16 \cdot 8 \mu \mathrm{m}$ in length. By TEM, bradyzoites had a very long $(10 \mu \mathrm{m})$ convoluted mitochondrion, up to 12 dense granules, but only 2 rhoptries. This redescription should help to differentiate the sarcocysts of $S$. fusiformis from similar sarcocysts in domestic and wild ruminants.
\end{abstract}

Key words: Sarcocystis fusiformis, sarcocyst, water buffalo (Bubalus bubalis), electron microscopy, ultrastructure.

\section{INTRODUCTION}

Species of Sarcocystis usually have a 2-host, preypredator life cycle, with herbivores as intermediate hosts and carnivores as definitive hosts (Dubey et al. 1989a). The intermediate host becomes infected with Sarcocystis species by ingesting sporocysts or oocysts, or both, excreted in the feces of the definitive host. After a brief period of schizogony, the parasite encysts in tissues and forms sarcocysts. The definitive host becomes infected by ingesting sarcocysts encysted in tissues of intermediate hosts.

Before the discovery of the life cycle of Sarcocystis species in 1972, only 1 species of Sarcocystis, Sarcocystis fusiformis, was recognized in cattle (Bos taurus) and the water buffalo (Bubalus bubalis) and it was thought to parasitize both host species. Subsequently, cattle were shown to have their own 3 species of Sarcocystis (Sarcocystis hirsuta, Sarcocystis hominis and Sarcocystis cruzi) and the name $S$. fusiformis was restricted to the large sarcocyst-forming species

* Corresponding author: USDA, ARS, APDL, BARCEast, Building 1001, Beltsville, MD 20705, USA. E-mail: jitender.dubey@ars.usda.gov in the water buffalo (Heydorn et al. 1975; Levine, 1977; Dubey et al. 1989a). Unlike cattle, life cycles including developmental stages of schizonts and sarcocysts are largely unknown for any species of the buffalo Sarcocystis.

Four valid species of Sarcocystis have been reported in the water buffalo: S. fusiformis, Sarcocystis buffalonis, Sarcocystis levinei and Sarcocystis dubeyi (Dissanaike and Kan, 1978; Huong et al. 1997a, b; Huong and Uggla, 1999; Huong, 1999a, b; Dubey et al. 2014). There is a debate whether the Sarcocystis species in cattle and buffaloes are the same species (Jehle et al. 2009; Chen et al. 2011; Gjerde, 2013; Dubey et al. 2014; Moré et al. 2014). However, there is general agreement that S. fusiformis of the water buffalo is distinctive and has no morphological equivalent in cattle. Although S. fusiformis is distinctive due to its shape and size, confusion remains regarding the ultrastructure of the sarcocyst and its contents. Most of the reports describing the ultrastructure of $S$. fusiformis are more than 30 years old and since then the electron microscopy technology has been much improved (Zaman and Colley, 1972; Ghaffar et al. 1978; Kan and Dissanaike, 1978; Zaman et al. 1980;

Parasitology, Page 1 of 10 . C Cambridge University Press 2014. This is a work of the U.S. Government and is not subject to copyright protection in the United States.

doi:10.1017/S003118201400122X 
Parairo et al. 1988; Dubey et al. 1989b; Wang et al. 1989). Here, we redescribe the structure of sarcocysts of $S$. fusiformis based on samples from water buffaloes in Egypt.

\section{MATERIAL AND METHODS}

\section{Naturally infected buffaloes}

Macroscopic S. fusiformis sarcocysts were collected immediately after slaughtering from the oesophagus of 4 buffaloes (5-8 years old) at El Warak slaughterhouse, Giza, Egypt in March 2014. Sarcocysts were fixed immediately in $2.5 \%$ glutaraldehyde in phosphate buffer ( $\mathrm{pH} 7 \cdot 2)$ and cut into $1 \mathrm{~mm}^{3}$ pieces each. Smears made from the sarcocyst contents were air dried, fixed with methanol and stained with Giemsa. The specimens were transported to the Faculty of Veterinary Science, University of Pretoria, Onderstepoort, South Africa on the same day of collection where they arrived $48 \mathrm{~h}$ later.

\section{Scanning electron microscopy}

Glutaraldehyde-fixed sarcocysts (GFS) were postfixed in $1 \%$ osmium tetroxide in Millonig's buffer ( $\mathrm{pH} 7 \cdot 4)$, dehydrated through a graded ethanol series into $100 \%$ ethanol. The absolute ethanol was substituted with liquid carbon dioxide and the samples critically point dried (E3100 Critical Point Drier, Quorum Technologies, Kent, UK), carbon coated and examined in a Zeiss Ultra Plus 55 FE-SEM (Carl Zeiss International, Oberkoch, Germany) operated at $1 \mathrm{kV}$.

\section{Transmission electron microscopy}

Twenty-one sarcocysts from 4 buffaloes were processed for TEM using standard techniques. Briefly, the GFS samples were post-fixed in $1 \%$ osmium tetroxide in Millonig's buffer ( $\mathrm{pH} 7 \cdot 4$ ), dehydrated through a series of graded ethanols, infiltrated with an epoxy resin/propylene oxide mixture before being embedded in absolute resin and polymerized at $60^{\circ} \mathrm{C}$ overnight. Ultrathin resin sections of macrocysts were contrasted with uranyl acetate and lead citrate and examined in a Philips CM10 TEM (FEI, Eindhoven, Netherlands) operated at $80 \mathrm{kV}$. Toluidine blue-stained resin sections were photographed with an Olympus BX63 compound microscope (Wirsam, Republic of South Africa).

\section{Abbreviations used for describing sarcocyst structures in text and in figures}

$\mathrm{am}=$ amylopectin granules, $\quad \mathrm{an}=$ anlagen, $\quad \mathrm{ar}=$ annular ring, $a p r=$ apical rings, $b r=$ bradyzoite, $\mathrm{co}=$ conoid, $\mathrm{cw}=$ cyst wall, $\mathrm{dg}=$ dense granule, $\mathrm{edl}=$ electron dense layer, gs $=$ ground substance, $\mathrm{hc}=$ host cell/tissue, $\mathrm{im}=$ inner membrane, $\mathrm{imt}=$ inner microtubules, $\mathrm{lp}=$ lipid bodies, $\mathrm{mb}=$ microtubules, $\mathrm{me}=$ metrocytes, $\mathrm{mn}=$ micronemes, $\mathrm{mp}=$ micropore, $\mathrm{mpc}=$ micropore collar, $\mathrm{mt}=$ mitochondrion, $\mathrm{nu}=$ nucleus, om $=$ outer membrane, $\mathrm{pe}=$ pellicle, $\mathrm{pl}=$ plasmalemma, $\mathrm{pp}=$ papillomatous structures, $\mathrm{pr}=$ polar rings, $\mathrm{pvm}=$ parasitophorous vacuolar membrane, $\mathrm{rh}=$ rhoptry, se $=$ septum, st $=$ subpellicular tubules, $\mathrm{vp}=$ villar protrusions.

\section{RESULTS}

\section{Macromorphology and light microscopy}

Sarcocysts of S. fusiformis were 3-10 mm long and 1-7 $\mathrm{mm}$ wide and could be easily separated from the host tissue (hc). They were typically fusiform in shape (wide in middle and tapered ends) but some appeared to have blunt ends (Fig. 1A). In routine $5 \mu \mathrm{m}$ histological sections stained with haematoxylin and eosin ( $\mathrm{H}$ and $\mathrm{E})$, the sarcocyst had a relatively thin $(2-5 \mu \mathrm{m}$ thick) smooth cyst wall (cw) that was not demarcated from the hc (Fig. 1B). In $1 \mu \mathrm{m}$ plasticembedded sections examined after staining with toluidine blue the sarcocyst wall stained faintly and had protrusions, like branches of a dead tree (Fig. 1C and D) and enclosed a thick layer of pale basophilic ground substance (gs) layer containing clusters of metrocytes (me). The interior of the sarcocyst was divided into round to oblong, closely packed divisions, by extensions of the gs. Bradyzoites in the central part of the sarcocyst were often degenerating. In smears stained with Giemsa, me were pink/ magenta in colour, round to oval in shape, $12 \cdot 3 \times$ $7 \cdot 3(9 \cdot 0-17 \cdot 5 \times 5 \cdot 0-8 \cdot 5, n=6) \mu \mathrm{m}$ in size and contained 2 or more nuclei (Fig. 1C, inset). The bradyzoites were elongated, $16.8 \times 4.3 \quad(14.5-$ $19 \cdot 0 \times 4 \cdot 0-4 \cdot 5, n=20) \mu \mathrm{m}$ in size. They had a vesicular subterminal nucleus (nu) and a darkly stained conoidal area (Fig. 1D, inset).

\section{Scanning electron microscopy}

The sarcocyst wall had a mesh-like (honey-comb) structure with irregularly shaped vp that were of different sizes and folded over the sarcocyst wall (Fig. 2A-F). The entire surfaces of vp were covered with papillomatous $(\mathrm{pp})$ structures that were nearly uniform in diameter $(85 \mathrm{~nm})$ at the tips (Fig. 2F). The total width of the $\mathrm{cw}$ in oblique sections appeared to be up to $9 \mu \mathrm{m}$.

The gs appeared granular and me were embedded in it (Fig. 2B and C). Surface view of me is shown in Fig. 2B and C. Bradyzoites were closely packed in groups, and groups were separated by thick septa (se) (Fig. 2C). The bradyzoites were $11.6 \times 2.7$ $(10 \cdot 5-12 \cdot 0 \times 2 \cdot 2-3 \cdot 0) \mu \mathrm{m}$ in size. Their surface was 

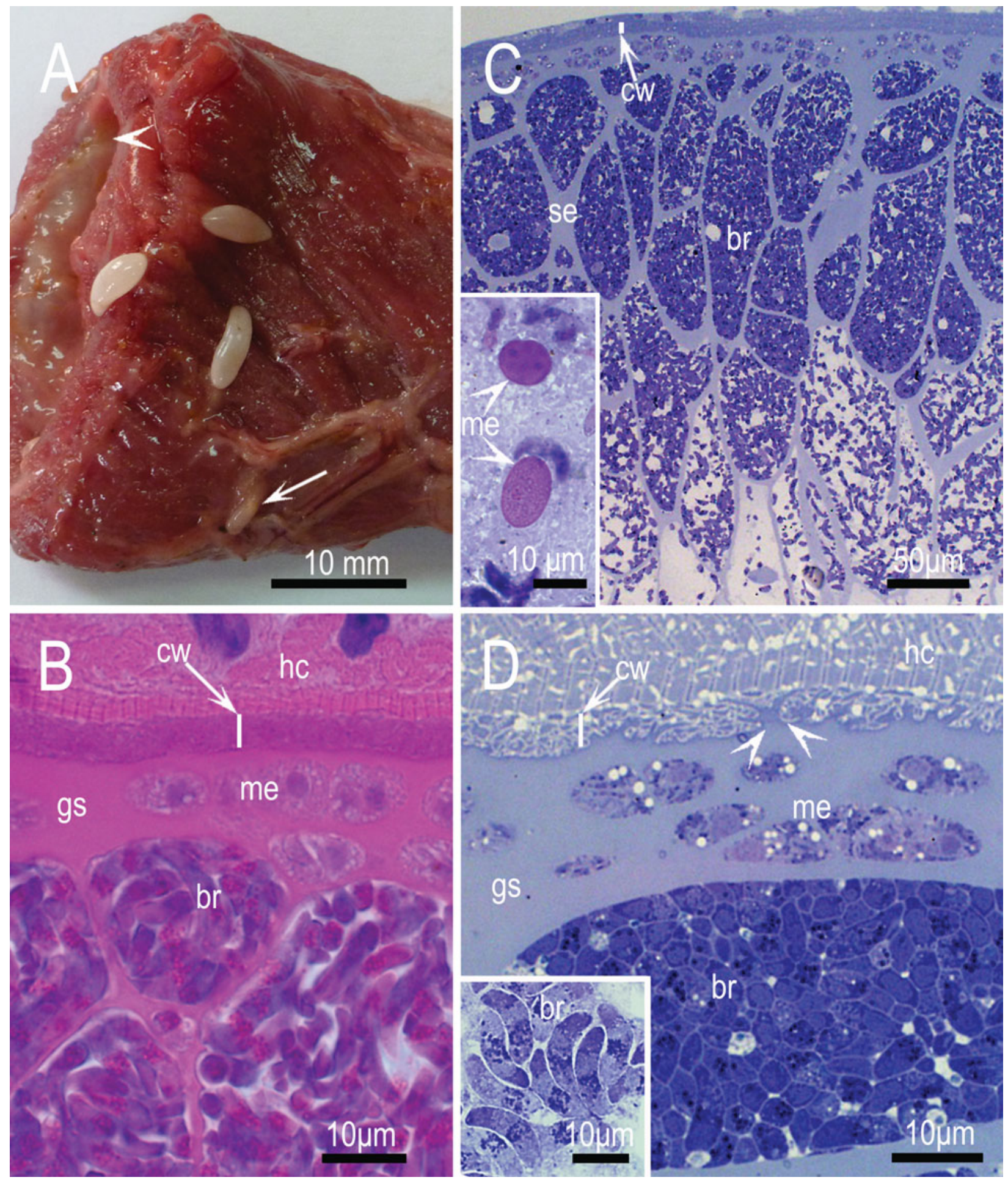

Fig. 1. Sarcocysts of S. fusiformis. (A) Four macrocysts in oesophagus of a buffalo, 1 of which is embedded in muscle (arrow); the oesophageal lumen is on the upper left-hand corner (arrowhead). Unfixed, unstained. (B) Sarcocyst in a $5 \mu \mathrm{m}$ section stained with $\mathrm{H}$ and $\mathrm{E}$. The cyst wall (cw) is not well demarcated from the host cell (hc). Note bradyzoites (br), and faintly staining metrocytes (me) in the ground substance (gs). (C) Sarcocyst in $1 \mu \mathrm{m}$ section stained with toluidine blue. Note thin cw and br packed in groups separated by septa (se). The br are packed tightly in peripheral zone and loosely arranged in interior of the sarcocyst. Inset shows two me in smear stained with Giemsa. (D) Higher magnification of the sarcocyst in (C). Note the cw is relatively thin, and villar protrusions (vp) are branched. Arrowheads point to branching of vp-like branches of a dead tree. The gs is homogeneous and approximately 5 times thicker than the cw. Metrocytes are located in the gs and a group of br are tightly packed. Inset shows several bradyzoites in smear stained with Giemsa. 

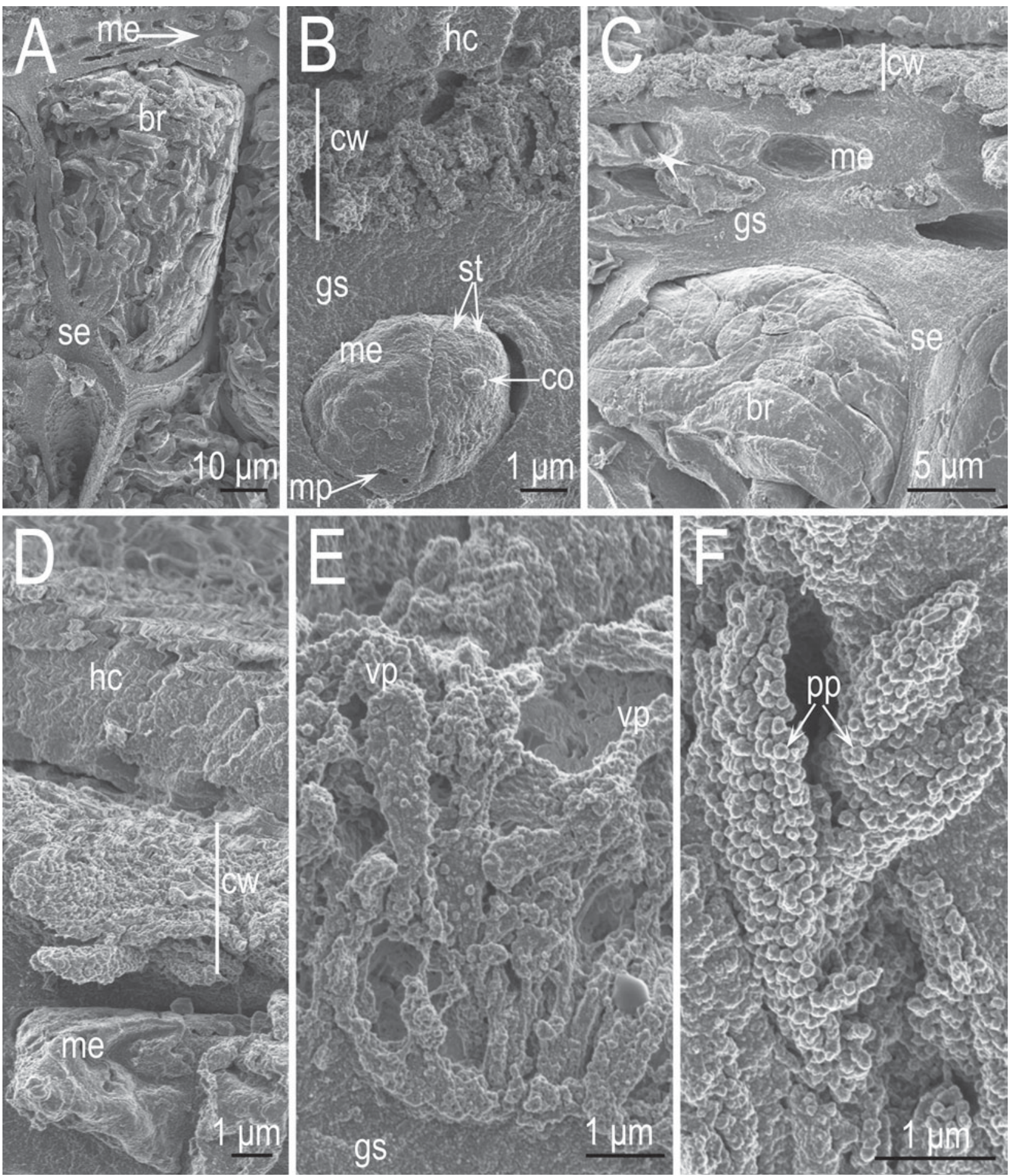

Fig. 2. SEM of S. fusiformis sarcocysts. (A) Sectioned part of a sarcocyst. Note bradyzoites (br) are arranged in sacks enclosed by thick septa (se). Metrocytes are located in the ground substance (gs); empty spaces represent location of fallen metrocytes (me) during processing. (B) Sectioned sarcocyst. Note host cell (hc) enclosing the cyst wall (cw), thick finely granular gs, and a single or group of me embedded in the gs. Part of outer covering of the me has ripped off showing a conoid (co), subpellicular tubules (st), and $\mathrm{mp}$. (C) Partly exposed sarcocyst. Note that villar protrusions are folded over the cw. The gs has several groups of me, 1 of which has 2 zoites (arrowhead). Also note a group of br enclosed in se. (D) Note combed appearance of the cw. (E) Higher magnification of the cw with irregularly shaped vp. (F) Higher magnification of the vp to show nearly identical papillomatous ( $p p$ ) structures on the vp.

uneven, and protuberances were more prominent in the non-conoidal half (Fig. 3). Subpellicular microtubules (st) originated from the papillary conoid (co) and extended throughout the bradyzoite (br) length; they were approximately $300 \mathrm{~nm}$ apart (Fig. 3B).

The micropore (mp) was located $3-4 \mu \mathrm{m}$ from the tip of the co (Fig. 3C). The mp had a rim, slightly 


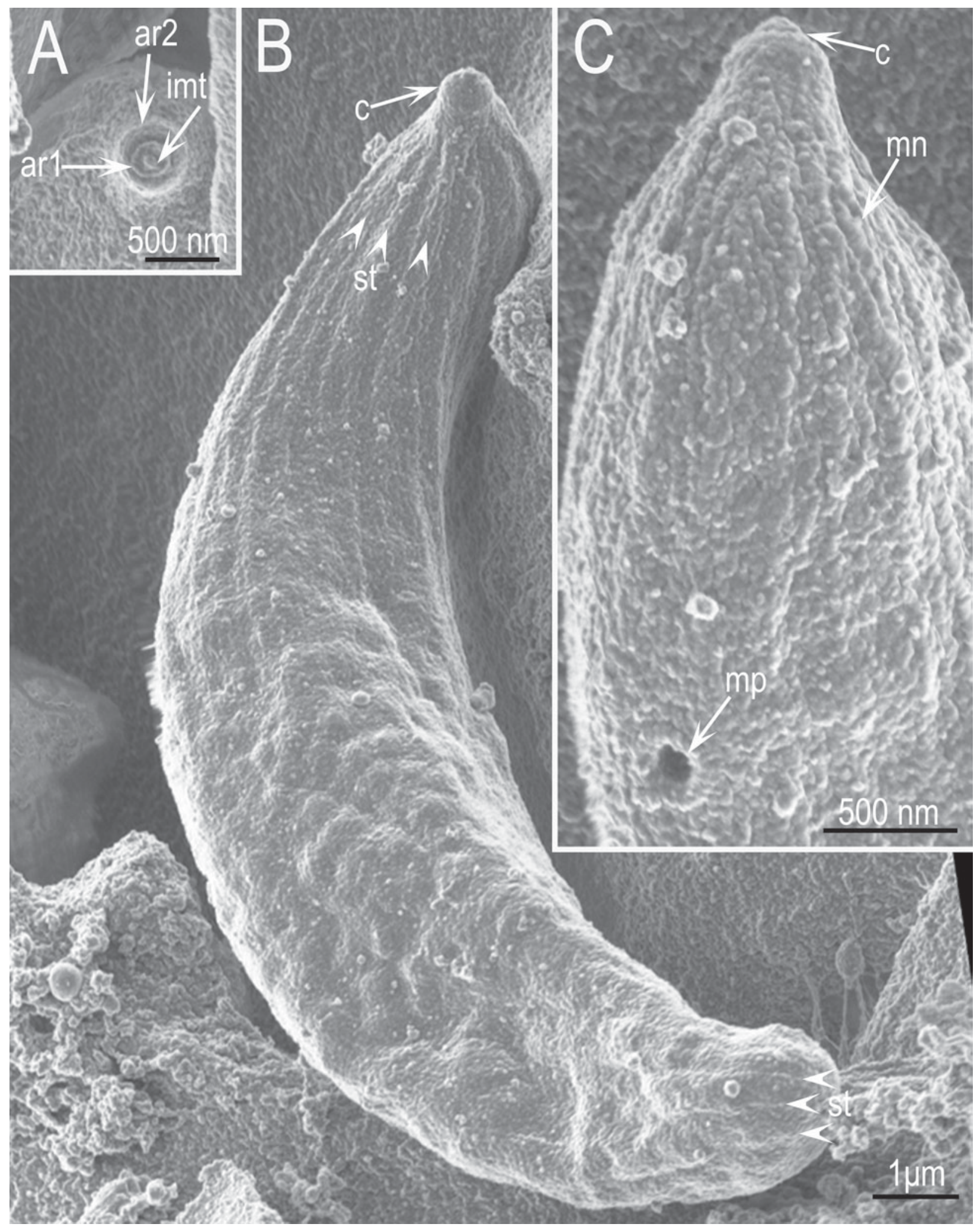

Fig. 3. SEM of bradyzoites. (A) Enface view of conoid (co). Note 2 inner microtubules (imt) and 2 apical rings (ar1, ar2). (B) Surface view of br. Note subpellicular tubules (st) arising from the polar ring (pr) of the co and extending to the entire length of the br. The posterior half of the bradyzoite (br) has irregular bumps. (C) Conoidal part of a br with a co and micropore $(\mathrm{mp})$; it is granular because of the numerous micronemes $(\mathrm{mn})$ that are partly visible through the pellicle (pe). 
raised from the surface of the br, and had a $200 \mathrm{~nm}$ pore (Fig. 3C). The micronemes $(\mathrm{mn})$ were visible through the pellicle (pe) (Fig. 3C).

\section{Transmission electron microscopy}

In total 21 sarcocysts were studied, the smallest being $3 \times 1 \mathrm{~mm}$ in size and the longest $1 \mathrm{~cm}$ long. Sarcocysts were structurally similar, irrespective of the size. The sarcocyst wall was not well demarcated from the hc; the total thickness of the sarcocyst wall (measured from the base of the vp to the area juxtaposed with hc) varied from $2 \cdot 0$ to $5 \cdot 2 \mu \mathrm{m}$ long within the same sarcocyst. The sarcocyst wall consisted of an outer most parasitophorous vacuolar membrane (pvm) that was lined by an electron dense layer (edl) that was up to $50 \mathrm{~nm}$ thick (Fig. 4C). The pvm was folded into branched vp. Some vp appeared to have a common stalk, like branches of a dead tree (Fig. 4B). These vp were up to $6 \mu \mathrm{m}$ long, but the exact length was difficult to determine because of branching (Fig. 4A-C). The vp contained filamentous tubular structures, most of which were parallel to the long axis of the projections; granules were absent from these tubules. Rarely a few mildly dense structures were present among longer tubules but these were probably cross-section of tubules; some tubules extended in to gs (Fig. 4C). The vp had out pocketing (evaginations) of mycelium or door stopper knobs at irregular distances (Fig. 4C). These knobs were up to $400 \mathrm{~nm}$ long and had a terminal end that was up to $100 \mathrm{~nm}$ thick and contained an edl. The edl of the cw extended along the length of the sarcocyst wall but was thin at areas of invaginations of the pvm (Fig. 4A-C). A relatively electron lucent gs layer was present immediately beneath the vp (Fig. 4A and B). The gs immediately below the pvm contained few granules, with indistinct boundaries (Fig. 4B). These granules were absent from the gs extending into the interior of the cyst.

Metrocytes, located in the gs, had relatively low electron density, compared with bradyzoites (Fig. 4A). They were globular to oblong in shape and $6-12 \mu \mathrm{m}$ long (Fig. 4A and D). They contained a nu, endoplasmic reticulum, lipid droplets, fissures, a few to several amylopectin granules and no $\mathrm{mn}$ or rhoptries.

Longitudinally cut bradyzoites measured from 3 sarcocysts were $14 \cdot 9(11 \cdot 2-16 \cdot 8, n=16) \mu \mathrm{m}$ long and $2 \cdot 5-4.0 \mu \mathrm{m}$ wide (Fig. 5). The length of the bradyzoites within the same sarcocyst varied from $11 \cdot 2$ to $16 \cdot 8 \mu \mathrm{m}$. It was difficult to find longitudinally cut bradyzoites (showing the co and the posterior end with $\mathrm{nu}$ ) because of their compactness in the sarcocyst. The bradyzoites had a double-membraned pe (an outer plasmalemma [pl], and an inner membrane [im], a co, $\mathrm{mn}, \mathrm{rh}$, amylopectin (am) and dense granules (dg), $\mathrm{mp}$, a mitochondrion $(\mathrm{mt})$ and a terminal nu (Figs. 5 and 6). The papillary co was truncated and up to $540 \times 400 \mathrm{~nm}$ in size. Sometimes electron dense material was present below the co (Fig. 6A). Micronemes were numerous and were dispersed throughout the anterior half, but were most abundant in the $1 / 3$ of the conoidal end of the parasite (Figs. 5 and 6). Micronemes were $285-358 \times 57-71 \mathrm{~nm}$ in size with tapering or round ends. Most mn were arranged in rows, but some were haphazardly arranged at the conoidal end (Fig. 6B). Some mn were present in the co (Fig. 6D). The electron density of mn varied; in some the central part was denser than the peripheral parts. The contents of rh had a varied electron density even within the same rh (Fig. 5B). Only 2 rh were seen in any 1 plane of section; the blind bulbous end extended up to the middle of the br. Amylopectin granules were numerous and dispersed in four-fifths of the non-conoidal end; up to 50 granules were seen in 1 plane of section. The single $\mathrm{mt}$ was convoluted, up to $10 \mu \mathrm{m}$ long, extending from the nu to the just posterior to micronemes (Fig. 5). The dg were $50-125 \mathrm{~nm}$ in diameter and located mostly in the middle part of bradyzoites. Micropores were up to $300 \times 200 \mathrm{~nm}$ in size and surrounded by granular electron dense material (Fig. 6D).

\section{Specimens deposited}

Voucher specimens of formalin fixed whole sarcocysts, histological sections stained with toluidine blue from the cases described are deposited in the US National Parasite Collection in the Division of Invertebrate Zoology and National Museum of Natural History, Smithsonian Institution, Washington, DC under (USNM):

- USNM 1250203, S. fusiformis, toluidine bluestained slide \#1

- USNM 1250204, S. fusiformis, toluidine bluestained slide \#2

- USNM 1250205, S. fusiformis, toluidine bluestained slide \#3

- USNM 1250206, S. fusiformis, toluidine bluestained slide \#4

- USNM 1250207, S. fusiformis, Giemsa-stained smear \#5

- USNM 1250208, S. fusiformis, Giemsa-stained smear \#6

- USNM 1250209, S. fusiformis, sarcocyst in formalin.

\section{DISCUSSION}

Sarcocysts of $S$. fusiformis are the largest in size for the genus. In the present study, sarcocysts were approximately $10 \mathrm{~mm}$ long. Kan and Dissanaike (1978) stated that sarcocysts from buffaloes in Malaysia were up to $25 \mathrm{~mm}$ long. To our knowledge the structure of microscopic immature sarcocysts of $S$. fusiformis is unknown. 

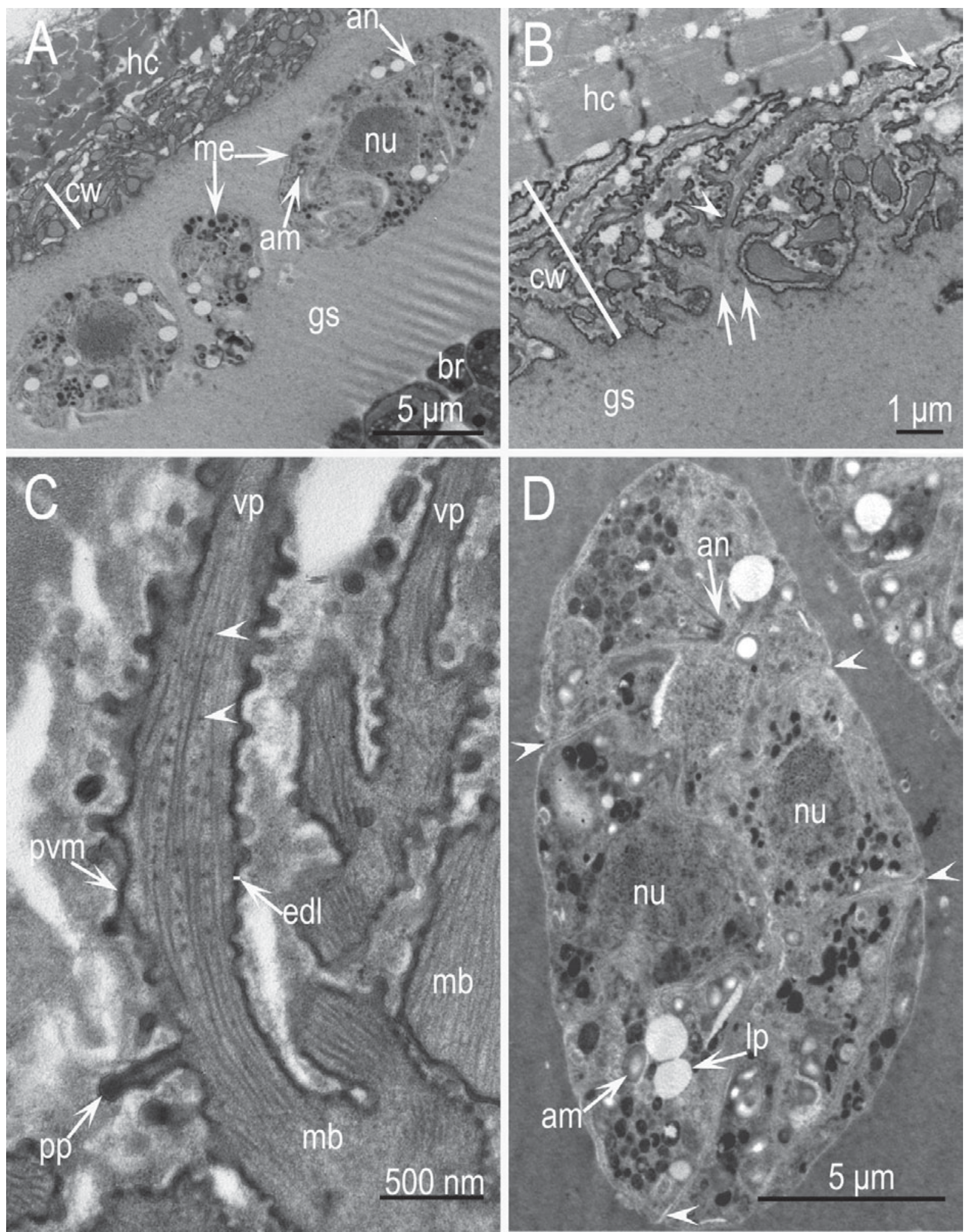

Fig. 4. TEM of $S$. fusiformis sarcocyst wall. The parasitophorous vacuolar membrane (pvm) is undulated with numerous villar protrusions (vp) with out-pocketing of protrusion ( $\mathrm{pp}$ ) branches with dumbbell-like ends. (A) The cyst wall $(\mathrm{cw})$ is poorly demarcated from the host cell (hc). Three metrocytes (me) are located in the ground substance (gs). The me on the far right has a daughter anlagen (an), few amylopectin (am), a large nucleus (nu), and numerous electron dense granules (dg)-like structures. Bradyzoites (bz) are located below gs. (B) Highly branched vp. Double arrows point to branching of vp, 1 of the vp is very long (arrowheads) and butted against the hc. The gs has few granules at the junction of vp. (C) Longitudinal view of a vp with longitudinally oriented microtubules (mb). Arrowheads point to few indistinct structures, probably $\mathrm{mb}$ cut in cross-section. Note dumbbell-like endings of pp structures. (D) A metrocyte with 2 nuclei (nu), several am, lipid bodies (lp), fissure-like structures (arrowheads), and absence of micronemes and rhoptries. Note zoite anlagen (an) with its conoid (co). Numerous un-identified electron dense structures of varying sizes are present. 


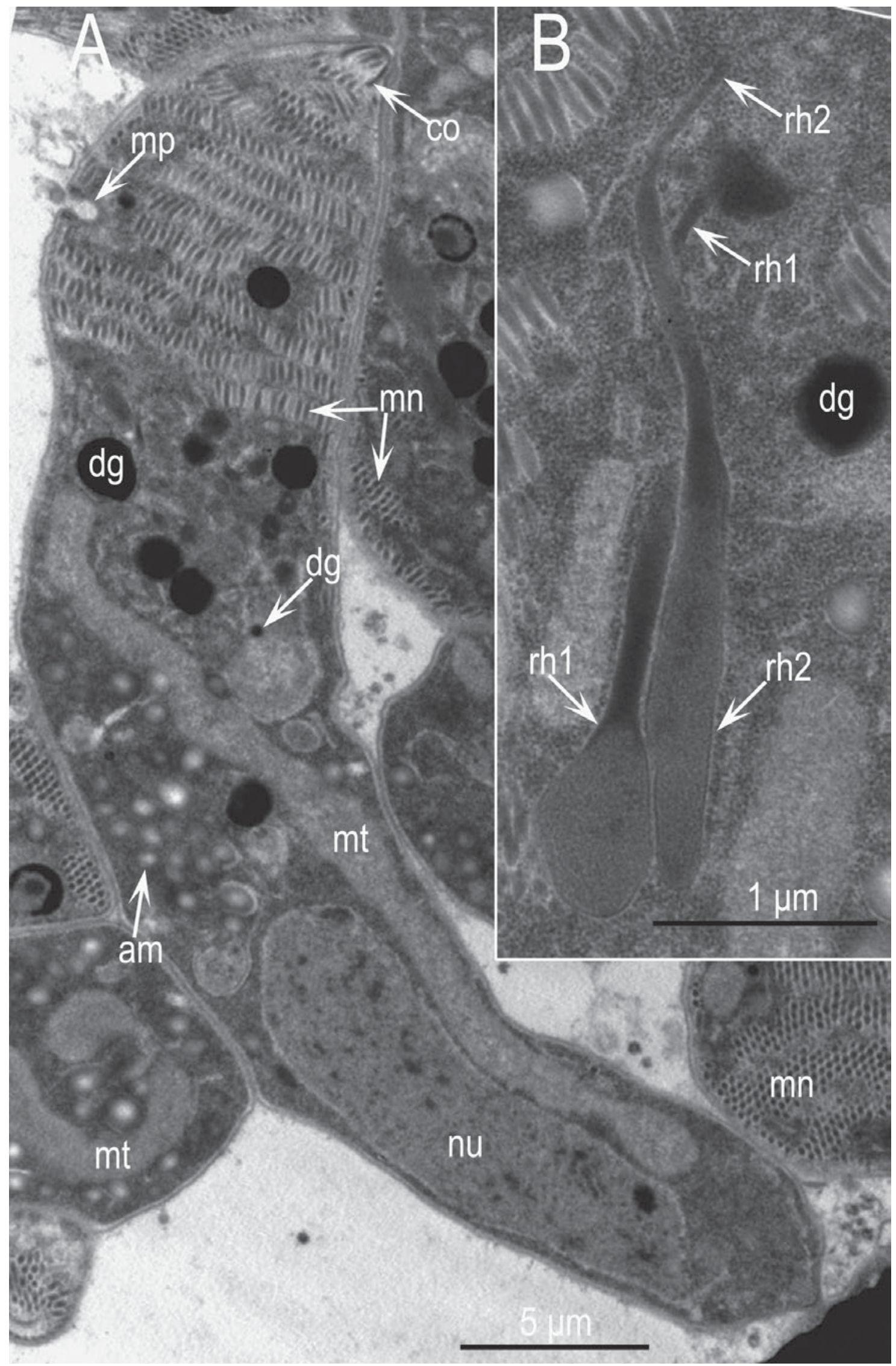

Fig. 5. TEM of bradyzoites. (A) A longitudinally cut bradyzoite (br) and parts of other br. Note conoid (co), numerous micronemes $(\mathrm{mn})$, a micropore $(\mathrm{mp})$, at least 8 dense granules $(\mathrm{dg})$ of different sizes, numerous amylopectin (am), a convoluted mitochondrion (mt) and a large nucleus (nu). (B) Part of a br showing 2 rhoptries (rh1, rh2) that are twisted at their necks. Note variability in density of the rh contents. 

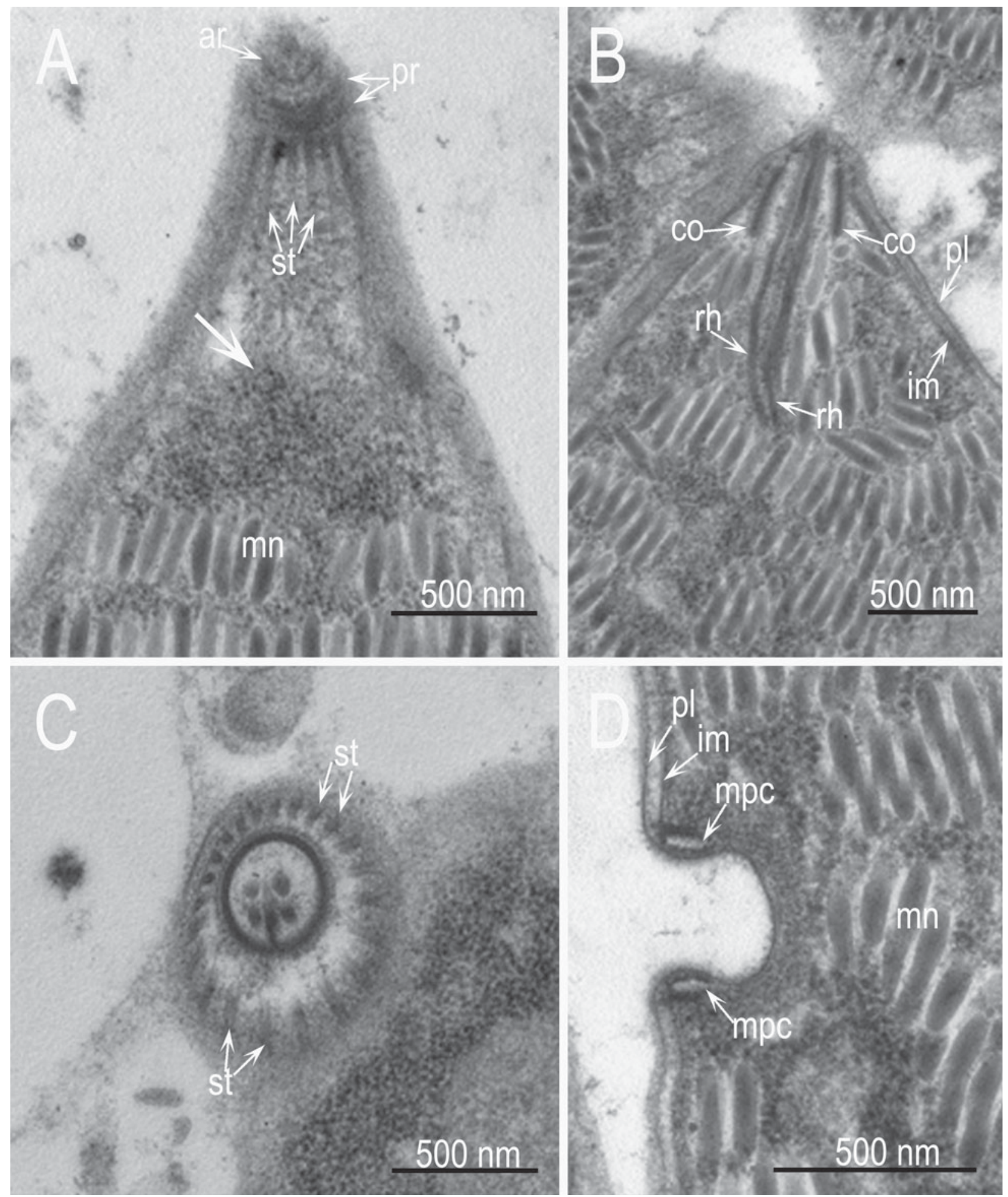

Fig. 6. TEM of conoidal part of 4 bradyzoites. (A) Protruding conoid (co) with annular (ar) and polar rings (pr), and subpellicular tubules (st) originating from the pr. Note granulated area (arrow) below co. The micronemes (mn) are arranged in rows. (B) Conoid with 2 rh opening in the co. Note haphazard arrangement of mn towards the conoidal end. (C) Cross/oblique-section through the co. Note 22 st originating from the pr. (D) Details of pellicle with outer plasmalemma ( $\mathrm{pl})$ and inner membrane (im) at the micropore $(\mathrm{mp})$ junction. The im is interrupted at the mp opening and collar/rim-like structure is present at the opening $(\mathrm{mpc})$. Electron dense secretory material surrounds the $\mathrm{mp}$. Note numerous mn.

In the present study, we examined sarcocysts by light microscopy, SEM and TEM. The only reference to SEM of $S$. fusiformis we found is that of Zaman et al. (1980). Their report is preliminary, but it illustrated that the bradyzoites are arranged in compartments, the bradyzoites have a micropore and several submicrotubules. Until now, the sarcocyst wall in S. fusiformis was described as cauliflower-like 
based on TEM. By SEM, the sarcocyst wall has a mesh-like (honey-comb) structure.

In all TEM studies with $S$. fusiformis there is general agreement that the sarcocyst wall is branched and has microtubules. Kan and Dissanaike (1978) were the first to distinguish $S$. fusiformis from $S$. levinei by TEM. The present study supplements their description of $S$. fusiformis. However, there is disagreement with respect to the structure of $\mathrm{mb}$ in the sarcocyst wall and rh in bradyzoites, and these differences might be related to fixation, and misinterpretations. Kan and Dissanaike (1978) and Dubey et al. (1989b) illustrated granules on mb that are similar to those in Fig. $4 \mathrm{C}$ in the present study. In our opinion these granules/structures were most probably part of cross-sections of the tubules.

In the present study, we provide details of S. fusiformis metrocytes, both by light microscopy and by electron microscopy. These varied in size and shape. Up to $3 \mathrm{mp}$ were seen (Fig. $2 \mathrm{~B}$ ), but it was not clear if they belonged to more than 1 metrocyte.

In previous TEM studies, several rh (10 according to Zaman and Colley (1972); 8 according to Kan and Dissanaike (1978); 8-13 according to Ghaffar et al. (1978); 8 according to Wang et al. (1989) were reported in $S$. fusiformis bradyzoites. Kan and Dissanaike (1978) described rh as uniformly dense without a limiting membrane. In the present study, we saw only $2 \mathrm{rh}$ in any br and the density of the rh contents varied within the same rh. It is most likely that in previous studies $\mathrm{dg}$ were misinterpreted as rh. Because the contents of rh can vary and simulate $\mathrm{dg}$, the presence of neck is necessary for their definitive identification.

Sarcocystis fusiformis sarcocysts are large in size and the density and arrangement of bradyzoites in the sarcocyst varies with their location in the sarcocyst; bradyzoites are more compact towards the peripheral part of the sarcocyst. Bradyzoites are curved, and hence difficult to measure and their size varies with the method of fixation and sectioning. In the present study, bradyzoites were shorter in sections $(11 \cdot 2$ $16 \cdot 8 \mu \mathrm{m})$ than in smears $(14 \cdot 5-19 \cdot 0 \mu \mathrm{m})$. In smears made in saline Kan and Dissanaike (1978) reported that bradyzoites were $16 \cdot 0-19 \cdot 4 \mu \mathrm{m}$. Bradyzoites are known to shrink after fixation and swell in size in saline. In the present study, bradyzoites in SEM appeared $1-4 \mu \mathrm{m}$ smaller than in TEM, probably related to drying during the preparation for SEM examination.

In conclusion, details of the structure of the $S$. fusiformis sarcocyst from the water buffalo as described here should form the basis for its differentiation from similar sarcocysts in other domestic and wild ruminants.

\section{ACKNOWLEDGEMENTS}

We would like to thank Dr C. A. Speer, regarding his advice for interpretation of ultrastructure.
FINANCIAL SUPPORT

R. Calero-Bernal is a postdoctoral fellow (ref. PO12010) funded by the Department of Employment and Innovation of the Regional Government of Extremadura (Spain) and the European Social Fund.

\section{REFERENCES}

Chen, X., Zuo, Y., Rosenthal, B. M., He, Y., Cui, L. and Yang, Z. (2011). Sarcocystis sinensis is an ultrastructurally distinct parasite of water buffalo that can cause foodborne illness but cannot complete its life-cycle in human beings. Veterinary Parasitology 178, 35-39.

Dissanaike, A.S. and Kan, S.P. (1978). Studies on Sarcocystis in Malaysia. I. Sarcocystis levinei n. sp. from the water buffalo Bubalus bubalis. Zeitschrift für Parasitenkunde (now Parasitology Research) 55, 127-138.

Dubey, J. P., Speer, C. A. and Fayer, R. (1989a). Sarcocystosis of Animals and Man, pp. 1-215. CRC Press, Boca Raton, FL, USA.

Dubey, J. P., Speer, C. A. and Shah, H. L. (1989b). Ultrastructure of sarcocysts from water buffalo in India. Veterinary Parasitology 34, 149-152. Dubey, J.P., Fayer, R., Rosenthal, B. M., Calero-Bernal, R. and Uggla, A. (2014). Identity of Sarcocystis species of the water buffalo (Bubalus bubalis) and cattle and the suppression of Sarcocystis sinensis as a nomen nudum. Veterinary Parasitology (in press). doi: 10.1016/ j.vetpar.2014.06.020

Ghaffar, F. A., Hilali, M. and Scholtyseck, E. (1978). Ultrastructural study of Sarcocystis fusiformis (Railliet,1897) infecting the Indian water buffalo (Bubalus bubalis) of Egypt. Tropenmedizin und Parasitologie 29, 289294.

Gjerde, B. (2013). Phylogenetic relationships among Sarcocystis species in cervids, cattle and sheep inferred from the mitochondrial cytochrome c oxidase subunit I gene. International fournal for Parasitology 43, 579-591. Heydorn, A. O., Gestrich, R., Mehlhorn, H. and Rommel, M. (1975). Proposal for a new nomenclature of the Sarcosporidia. Zeitschrift für Parasitenkunde (now Parasitology Research) 48, 73-82.

Huong, L. T. T. (1999a). Sarcocystis infections of the water buffalo in Vietnam. Doctoral thesis. Swedish University of Agricultural Sciences, Uppsala, Sweden, pp. 1-53.

Huong, L. T. T. (1999b). Prevalence of Sarcocystis spp. in water buffaloes in Vietnam. Veterinary Parasitology 86, 33-39.

Huong, L. T. T. and Uggla, A. (1999). Sarcocystis dubeyi n. sp. (Protozoa: Sarcocystidae) in the water buffalo (Bubalus bubalis). Fournal of Parasitology 85, 102-104.

Huong, L. T. T., Dubey, J.P., Nikkilä, T. and Uggla, A. (1997a). Sarcocystis buffalonis $\mathrm{n}$. sp. (Protozoa: Sarcocystidae) from the water buffalo (Bubalus bubalis) in Vietnam. Fournal of Parasitology 83, 471-474.

Huong, L. T. T., Dubey, J. P. and Uggla, A. (1997b). Redescription of Sarcocystis levinei Dissanaike and Kan, 1978 (Protozoa: Sarcocystidae) of the water buffalo (Bubalus bubalis). Fournal of Parasitology 83, 1148-1151. Jehle, C., Dinkel, A., Sander, A., Morent, M., Romig, T., Luc, P. V., De, T.V., Thai, V.V. and Mackenstedt, U. (2009). Diagnosis of Sarcocystis spp. in cattle (Bos taurus) and water buffalo (Bubalus bubalis) in northern Vietnam. Veterinary Parasitology 166, 314-320.

Kan, S. P. and Dissanaike, A. S. (1978). Studies on Sarcocystis in Malaysia II. Comparative ultrastructure of the cyst wall and zoites of Sarcocystis levinei and Sarcocystis fusiformis from the water buffalo, Bubalus bubalis. Zeitschrift für Parasitenkunde (now Parasitology Research) 57, 107-116.

Levine, N. D. (1977). Nomenclature of Sarcocystis in the ox and sheep and of fecal coccidia of the dog and cat. Fournal of Parasitology 63, 36-51.

Moré, G., Pantchev, A., Skuballa, J., Langenmayer, M. C., Maksimov, P., Conraths, F. J., Venturini, M. C. and Schares, G. (2014). Sarcocystis sinensis is the most prevalent thick-walled Sarcocystis in beef for consumers in Germany. Parasitology Research 113, 2223-2230.

Parairo, J. R., Manuel, M. F. and Icatlo, F. C. (1988). Ultrastructural studies of the cyst wall of Sarcocystis spp. In Philippine carabaos. Philippine Fournal of Veterinary and Animal Science 14, 40-54.

Wang, M., Liu, H.H., Lin, Q.W., Xiao, B. N., Zhang, C. G. and Gong, Z. F. (1989). A comparative ultrastructural study on the two types of sarcocysts in Chinese buffaloes. Acta Veterinaria et Zootechnica Sinica 20, 356-362.

Zaman, V. and Colley, F. C. (1972). Fine structure of Sarcocystis fusiformis from the Indian water buffalo (Bubalus bubalis) in Singapore. Southeast Asian Yournal of Tropical Medicine and Public Health 3, 489-495.

Zaman, V., Robertson, T. A. and Papadimitriou, J. M. (1980). Scanning electron microscopy of Sarcocystis fusiformis from the water buffalo (Bubalus bubalis). Southeast Asian Fournal of Tropical Medicine and Public Health 11, 205-211. 